\title{
Estrategias para mejorar el rendimiento académico de la asignatura de matemáticas
}

Estratégias para melhorar o desempenho acadêmico da disciplina de matemática

Strategies to improve the academic performance of the subject of mathematic

\section{Sully T. Muñoz}

Ph.D. Universidad Nacional de Colombia, Bogota, Colombia. https://orcid.org/0000-00032998-2717 sullytigreros@gmail.com

Recibido octubre 2019 - Aceptado mayo 2020

Formación docente - revista iberoamericana de educación

http://www.revista-iberoamericana.org/index.php/es/index https://creativecommons.org/licenses/by/4.0/deed.es

e-ISSN: 2737-632X

Vol - 3 No. 3 julio - septiembre 2020

Pags 33-52

Resumen El tema y objetivo de este artículo ha tenido como propósito fundamental establecer la aplicación de las estrategias didácticas que se deben emplear en la enseñanza aprendizaje en la asignatura de matemáticas de la Carrera de Electrónica y Telecomunicaciones de la Universidad Estatal Península de Santa Elena, La Libertad. De acuerdo a un análisis se detectó que en el 2008 se produjo un 38\% de estudiantes reprobados, en el 2009 el $28 \%$ de estudiantes reprobados y en el 2010 el $71 \%$ de estudiantes reprobados en la carrera antes mencionada.

Palabras clave: Pedagogía Teoría científica, metodología, rendimiento

Abstract. The theme and purpose of this article has as main purpose to establish the application of the teaching strategies to be used in teaching and learning in the mathematics of the School of Electronics and 
Telecommunications of the Santa Elena Peninsula State University, La Libertad. According to an analysis it found that in 2008 there was a $38 \%$ of students fail the test, in $200928 \%$ of students retained and in $201071 \%$ of students fail the test in the aforementioned race.

Key words: Higher Education, Graduates, Professionals, Entrepreneurs,

Resumo O principal objetivo e objetivo deste artigo foi estabelecer a aplicação de estratégias didáticas a serem utilizadas no ensino e aprendizagem na disciplina de matemática do curso de Eletrônica e Telecomunicações da Universidade Estadual da Península Santa Elena, La Libertad. . De acordo com uma análise, foi detectado que em 2008 havia 38\% de alunos reprovados, em $200928 \%$ de alunos reprovados e em $201071 \%$ de alunos reprovados no grau mencionado.

Palavras-chave: Ensino Superior, Graduados, Profissionais, Empreendedores.

\section{INTRODUCCIÓN}

La finalidad que originó la publicación de esta primera edición fue justamente servir de apoyo a estudiantes y profesionales de algunas disciplinas, para que puedan desenvolverse adecuadamente en los procesos y complejos de temas de las matemáticas en lo relacionado a estrategias didácticas de enseñanza y aprendizaje. En esta edición se hace un análisis profundo de tres periodos académicos, donde se verifica falencias y bajo rendimiento en tres etapas consecutivas donde se verificaba pérdida de año desde un $38 \%$ hasta $71 \%$, de las Estrategias didácticas se analizan un conjunto de procedimientos que apoyados de técnicas de enseñanza tienen por objeto llevar a buen término la acción didáctica, es decir alcanzar los objetivos de aprendizaje y por lo tanto 
obtener un buen rendimiento académico en la asignatura de matemáticas, se decide abordarlas bajo la intención de conocer si se aplican o no en las aulas universitarias. El estudio de la matemática en la Educación Superior se integra a un mundo cambiante, complejo e incierto. Cada día aparece nueva información, nuevas teorías, nuevas formas de entender la vida y distintas maneras de interacción social. La matemática es una forma de aproximación a la realidad, brinda elementos de importancia para el proceso vital y permite a la persona entenderla y, más aún, transformarla, porque en su nivel más elemental, responde a inquietudes prácticas: la necesidad de ordenar, cuantificar y crear un lenguaje para las transacciones comerciales. La mayoría de los estudiantes de la carrera de Electrónica y Telecomunicaciones, en la materia de matemáticas de la universidad Estatal Península de Santa Elena, se encuentran con un grave problema al ingresar al pre universitario, al no contar con los suficientes conocimientos para afrontar los nuevos cambios.

Siendo sus guías los profesores deben tomar conciencia en cuanto a la capacitación que se debe tener para realizar una buena planificación no basta sólo con los conocimientos adquiridos en una universidad o en un instituto, deben buscar la manera de solventar las dificultades, afianzando la práctica pedagógica hacia el constructivismo. Estas son las causas por las cuales, esta propuesta está dirigida a una capacitación docente, evaluación de desempeño, elaboración y aplicación de estrategias didácticas para una enseñanza de calidad que permitan el mejoramiento del rendimiento académico de los estudiantes en la materia de matemáticas evitando así que año tras año se vuelva un problema para los que ingresen al pre universitario.

Sin duda las matemáticas es una herramienta de vital importancia para la vida práctica de todos y aplicar un instrumento que permita valorar la capacidad 
de los estudiantes en la solución de problemas matemáticos, va a permitir que ellos alcancen las metas planteadas.

Podríamos definir a las estrategias de enseñanza como los procedimientos o recursos utilizados por el agente de enseñanza para promover aprendizajes significativos (Mayer, 1984; Shuell, 1988; West, Farmer y Wolff, 1991). La investigación de estrategias de enseñanza ha abordado aspectos como los siguientes: diseño y empleo de objetivos e intenciones de enseñanza, preguntas insertadas, ilustraciones, modos de respuesta, organizadores anticipados, redes semánticas, mapas conceptuales y esquemas de estructuración de textos, entre otros (Díaz Barriga y Lule, 1978). Las estrategias seleccionadas han demostrado, su efectividad al ser introducidas como apoyos en textos académicos así como en la dinámica de la enseñanza (exposición, negociación, discusión, experiencia, demostración, proyectos, etc.) ocurrida en la clase. Las principales estrategias de enseñanza son las siguientes.

- Resúmenes

- Ilustraciones

- Organizadores previos

- Preguntas intercaladas

- Mapas conceptuales y redes semánticas

- Uso de estructuras textuales.

Diversas estrategias de enseñanza pueden incluirse antes (preinstruccionales), durante (coinstruccionales) o después (posinstruccionales) de un contenido curricular específico, ya sea en un texto o en la dinámica del trabajo docente. En ese sentido podemos hacer una primera clasificación de 
las estrategias de enseñanza, basándonos en su momento de uso y presentación.

Las estrategias pre-instruccionales por lo general preparan y alertan al estudiante en relación a qué y cómo va a aprender (activación de conocimientos y experiencias previas pertinentes), y le permiten ubicarse en el contexto del aprendizaje pertinente. Algunas de las estrategias preinstruccionales típicas son: los objetivos y el organizador previo. Las estrategias coinstruccionales apoyan los contenidos circulares durante el proceso mismo de enseñanza. Cubren funciones como las siguientes: detección de la información principal; conceptualización de contenidos; delimitación de la organización, estructura e interrelaciones entre dichos contenidos, y mantenimiento de la atención y motivación. Aquí pueden incluirse estrategias como: ilustraciones, redes simétricas mapas conceptuales entre otros. A su vez las estrategias posinstruccionales se presentan después del contenido que se ha de aprender, y permitan al alumno formar una visión sintética, integradora e incluso crítica del material. Entre otros casos le permiten valorar su propio aprendizaje. Algunas de las estrategias posinstruccionales más reconocidas son: preguntas intercaladas, resúmenes finales, redes semánticas y mapas conceptuales.

Otra clasificación valiosa puede ser desarrollada a partir de los procesos cognitivos que las estrategias explicitan para promover mejores aprendizajes. De otro modo, una segunda clasificación se debería considerar como sigue:

- Estrategias para activar conocimientos previos y para establecer expectativas adecuadas en los alumnos.

- Estrategias para orientar la atención de los alumnos.

- Estrategia para organizar la información que se ha de aprender. 
- Estrategias para promover entre los conocimientos previos y la nueva información que se ha de aprender.

Que para definir la literatura o los métodos, se tengan presente algunas diferenciaciones que emergen de los diferentes niveles del Sistema Educativo.

Como estudio de los métodos, en la Pedagogía Universitaria pone en juego, el estudio de los métodos propios de las ciencias y los métodos pedagógicos, para de allí derivar caminos posibles en el quehacer docente.

Coordina las actividades encaminadas a garantizar el proceso de enseñanza aprendizaje de una unidad didáctica. De esta manera los criterios metodológicos dan el sentido pedagógico a la estrategia que mediante su planificación garantiza procesos coherentes para las actividades de desarrollo de la competencia. Una estrategia de aprendizaje es un procedimiento (conjunto de pasos o habilidades) que un alumno adquiere y emplea de forma intencional como instrumento flexible para aprender significativamente y solucionar problemas y demandas académicas. Las estrategias de aprendizaje son ejecutadas intencionalmente por un aprendiz siempre que le demande aprender, recordar o solucionar problemas sobre algún contenido de aprendizaje. Cuando un sistema educativo decide establecer un proceso de evaluación del desempeño profesional del docente, la primera pregunta que debe hacerse es: "para qué evaluar". Se trata de un asunto delicado, entre otras, por las siguientes razones:

- Por las inquietudes que despierta un proceso de este tipo.

- Por los efectos secundarios que puede provocar.

- Por problemas éticos.

- Acerca de las inquietudes que suele despertar un proceso de este tipo. 
Los profesores, en principio, se resisten a ser evaluados. Un planteamiento apresurado, acompañado de un estado de desinformación o una información sesgada pueden disparar las especulaciones, creencias y suposiciones erróneas, interesadas o malintencionadas y provocar una oleada de protestas y resistencia activa, tanto de ellos como sus organizaciones sindicales y profesionales, que truncarán toda posibilidad de procesos útiles para la mejora.

Una buena evaluación profesoral debe cumplir las funciones siguientes:

- Función de diagnóstico

- Función instructiva

- Función educativa

- Función desarrolladora

El carácter desarrollador de la evaluación del maestro se cumple también cuando la misma contiene juicios sobre lo que debe lograr el docente para perfeccionar su trabajo futuro, sus características personales y para mejorar sus resultados

La educación es un hecho intencionado y, en términos de calidad de la educación, todo proceso educativo busca permanentemente mejorar el rendimiento del estudiante. En este sentido, la variable dependiente clásica en cualquier análisis que involucra la educación es el rendimiento académico, el cual es definido de la siguiente manera: "Del latín reddere (restituir, pagar) el rendimiento es una relación entre lo obtenido y el esfuerzo empleado para obtenerlo. Es un nivel de éxito en la universidad, en el trabajo, etc.", El problema del rendimiento académico se entenderá de forma científica cuando se encuentre la relación existente entre el trabajo realizado por los profesores 
y los estudiantes, de un lado, y la educación (es decir, la perfección intelectual y moral lograda por éstos) de otro, al estudiar científicamente el rendimiento, es básica la consideración de los factores que intervienen en él. Por lo menos en lo que a la instrucción se refiere, existe una teoría que considera que el buen rendimiento académico se debe predominantemente a la inteligencia de tipo racional; sin embargo, lo cierto es que ni siquiera en el aspecto intelectual del rendimiento, la inteligencia es el único factor. Al analizarse el rendimiento académico, deben valorarse los factores ambientales como la familia, la sociedad, las actividades extracurriculares y el ambiente estudiantil, los cuales están ligados directamente con nuestro estudio del rendimiento académico.

Además el rendimiento académico es entendido como una medida de las capacidades respondientes o indicativas que manifiestan, en forma estimativa, lo que una persona ha aprendido como consecuencia de un proceso de instrucción o formación. De la misma forma, ahora desde una perspectiva propia del estudiante, se define el rendimiento como la capacidad de responder satisfactoriamente frente a estímulos educativos, susceptible de ser interpretado según objetivos o propósitos educativos pre-establecidos. Este tipo de rendimiento académico puede ser entendido en relación con un grupo social que fija los niveles mínimos de aprobación ante un determinado grupo de conocimientos o aptitudes. Según Herán y Villarroel (1987), el rendimiento académico se define en forma operativa y tácita afirmando que se puede comprender el rendimiento previo como el número de veces que el estudiante ha repetido uno o más cursos. Después de realizar un análisis comparativo de diversas definiciones del rendimiento académico, se puede concluir que hay un doble punto de vista, estático y dinámico, que encierran 
al sujeto de la educación como ser social. En general, el rendimiento académico es caracterizado del siguiente modo:

a) El rendimiento en su aspecto dinámico responde al proceso de aprendizaje, como tal está ligado a la capacidad y esfuerzo del alumno;

b) En su aspecto estático comprende al producto del aprendizaje generado por el estudiante y expresa una conducta de aprovechamiento;

c) El rendimiento está ligado a medidas de calidad y a juicios de valoración;

d) El rendimiento es un medio y no un fin en sí mismo;

e) El rendimiento está relacionado a propósitos de carácter ético que incluye expectativas económicas, lo cual hace necesario un tipo de rendimiento en función al modelo social vigente.

En consonancia con esa caracterización y en directa relación con los propósitos de la investigación, es necesario conceptuar el rendimiento académico. Para ello se requiere previamente considerar dos aspectos básicos del rendimiento: el proceso de aprendizaje y la evaluación de dicho aprendizaje. El proceso de aprendizaje y las teorías que tratan los procesos de adquisición de conocimiento han tenido durante este último siglo un enorme desarrollo debido fundamentalmente a los avances de la psicología y de las teorías instruccionales, que han tratado de sistematizar los mecanismos asociados a los procesos mentales que hacen posible el aprendizaje [Reigeluth, 1983].

El propósito de las teorías educativas es el de comprender e identificar estos procesos y a partir de ellos, tratar de describir métodos para que la instrucción 
sea más efectiva. Es en este último aspecto en el que principalmente se basa el diseño instruccional, que se fundamenta en identificar cuáles son los métodos que deben ser utilizados en el diseño del proceso de instrucción, y también en determinar en qué situaciones estos métodos deben ser usados.

De acuerdo con [Reigeluth, 1987], de la combinación de estos elementos (métodos y situaciones) se determinan los principios y las teorías del aprendizaje. Un principio de aprendizaje describe el efecto de un único componente estratégico en el aprendizaje de forma que determina el resultado de dicho componente sobre el enseñante bajo unas determinadas condiciones. Desde el punto de vista prescriptivo, un principio determina cuándo debe este componente ser utilizado. Por otro lado, una teoría describe los efectos de un modelo completo de instrucción, entendido como un conjunto integrado de componentes estratégicos en lugar de los efectos de un componente estratégico aislado.

A este respecto, el estudio de la mente y de los mecanismos que intervienen en el aprendizaje se ha desarrollado desde varios puntos de vista basados en la misma cuestión fundamental, a saber: ¿Cuáles son las condiciones que determinan un aprendizaje más efectivo? [Gagné, 1987].

Sobre la evaluación académica hay una variedad de postulados que pueden agruparse en dos categorías: aquellos dirigidos a la consecución de un valor numérico (u otro) y aquellos encaminados a propiciar la comprensión en términos de utilizar también la evaluación como parte del aprendizaje. En el presente trabajo interesa la primera categoría, que se expresa en los calificativos universitarios. Las calificaciones son las notas o expresiones cuantitativas o cualitativas con las que se valora o mide el nivel del rendimiento académico en los estudiantes. Las calificaciones son el resultado 
de los exámenes o de la evaluación continua a que se ven sometidos los estudiantes. Medir o evaluar los rendimientos es una tarea compleja que exige del docente obrar con la máxima objetividad y precisión.

En el sistema educativo ecuatoriano, en especial en las universidades, la mayor parte de las calificaciones se basan en el sistema decimal, es decir de 0 a 10. Sistema en el cual el puntaje obtenido se traduce a la categorización del logro del aprendizaje.

\section{MATERIALES Y MÉTODOS}

La metodología empleada para el desarrollo de la presente investigación, es la expresada por Hernández Sampieri (2010), evidenciada en su libro Metodología de la Investigación, en éste capítulo se abordan tópicos como el alcance, enfoque y diseño de la investigación, la selección, tipo y tamaño de muestra, cómo se realizó la recolección de los datos, el instrumento de medición y el análisis de los resultados Al definir esta conceptualización se puede interiorizar las estrategias de investigación, el diseño, los procedimientos, serán distintos en cada uno de los alcances. No obstante cualquier investigación, puede incluir "elementos" de más de uno de estos cuatro alcances, los mismos que obedecen a la siguiente nomenclatura: Exploratorios, Descriptivos, Correlacionales y Explicativos.

Los estudios descriptivos, evidencian una situación o realidad tal como se presentan, por medio de estos estudios se definen las propiedades, características o géneros explicativos de los fenómenos sometidos al análisis investigativo, es decir, que en los estudios descriptivos, se procede a la 
"fotografía" del evento, tal como se presenta, sin intervenir el investigador de alguna manera a modificar alguna variable de estudio, lo que implica que no se produce inferencia sobre los resultados, o sobre el proceso para la obtención de la información técnica.

De la misma forma que los estudios exploratorios pretenden Graficar y descubrir, los descriptivos se centran en recolectar datos que muestren un evento, una comunidad, un fenómeno, hecho, contexto o situación que ocurre, en diferentes aspectos, por ello es fundamental que el investigador esté en capacidad de definir qué variables se medirán o sobre qué se tomarán los datos, esto no implica necesariamente, que en el devenir de la investigación surjan nuevos tópicos sobre los cuales se requiera el levantamiento técnico de información.

Los estudios correlacionales, pretenden evaluar el nivel de relación que existe entre una o más variables de estudio, es decir agrupa las variables de estudio, mediante un patrón predecible para la muestra o la población. Los estudios correlacionales son de mucha utilidad en la comprobación de una variable al identificar el comportamiento de otras variables vinculadas, lo que implica que si dos variables se encuentran correlacionadas y se determina el nivel de la asociación, se tienen bases para predecir con un grado apreciable de aceptación, el valor aproximado que tiene un grupo de personas en una variable, al identificar qué valor se expresa en otra.

Los estudios explicativos tienen por propósito, determinar por qué suceden los eventos o fenómenos, es decir pretende explicar los hechos, a partir de la observación y relación causa-efecto. 
En el marco de ésta investigación, se consideró oportuno realizar un estudio detallado de las estrategias didácticas como parte de la metodología del docente que permiten el mejoramiento del rendimiento académico de los estudiantes del pre universitario en la asignatura de matemáticas de la Universidad Estatal Península de Santa Elena en particular a la Carrera de Electrónica y Telecomunicaciones.

Con estos propósitos y estos supuestos se presenta a continuación las etapas de investigación puntualizando por medio de sus objetivos los temas centrales.

Esta investigación inicia con carácter descriptivo, pues se ha llegado a determinar la situación de las variables involucradas en el estudio en un momento dado con relación a su presencia o ausencia, la frecuencia con que se presenta un fenómeno (incidencia o prevalencia), características de las personas, lugar y periodo donde ocurre. Procediendo a la observación de los hechos tal como ocurren con el objeto de describirlos, posteriormente se desarrolla con alcance correlacional, al pretender predecir comportamientos de los involucrados, a partir de la correlación de las variables de estudio.

El enfoque cuantitativo es un proceso riguroso, secuencial y sistematizado en el que se busca resolver problemas, bien sea de vacío de conocimiento (investigación científica) o de gerencia, pero en ambos casos es organizado y garantiza la producción de conocimiento o de alternativas de solución viables, empleando para el efecto pruebas estadísticas en función de determinar la relación de las variables de estudio.

Las variables que se aplican en la investigación cuantitativa son: 
- Variables cuantitativas continuas: cuando entre dos valores, son posibles infinitos valores intermedios. Por ejemplo, edad, talla, etc.

- Variables cuantitativas discontinuas o discretas. Asumen sólo valores enteros. Por ejemplo, números de alumnos por aula, etc.

Hernández et al (2010), indica que el proceso cuantitativo implica 10 fases, las mismas, que inician con la idea de la investigación y culmina con la presentación del Informe final, por tanto, podemos expresar que el presente trabajo investigativo, se contextualiza dentro del enfoque cuantitativo.

Para el levantamiento técnico de información se procedió a la elaboración de encuestas para estudiantes y docentes por constituir la herramienta que brinda más facilidades para la recopilación de datos primarios, así como una entrevista y la evaluación de desempeño. La ventaja principal del método de encuesta es su flexibilidad. Se puede usar para obtener muchas clases de información, en situaciones diferentes. Dependiendo del diseño de la encuesta, también puede proporcionar información con mayor rapidez y a un costo más bajo que la investigación mediante la observación. Para el estudio se empleó un modelo de encuesta.

\section{RESULTADOS}

Una vez obtenidos los valores de las varianzas, se procede a la aplicación de la fórmula para la determinación del índice de Confiabilidad Alfa de Cronbach. Como puede observarse, se ubica el número de ítems, que en nuestro caso ha sido de 18 preguntas seleccionadas de las 23 planteadas en el instrumento de medición. Adicionalmente, la varianza total del instrumento, es decir, la suma de las varianzas de los ítems (8.7705) se emplea como 
numerador, y la varianza total de los ítems (30.0516), como denominador. Con esta aplicación se obtiene un índice de confiabilidad de 0.75 , éste procedimiento permite interpretar que el instrumento empleado para el levantamiento técnico de información, cuenta con un coeficiente de confiabilidad del $75 \%$ y, empleando la clasificación proporcionada por George y Mallery (1995) citado por Hernández et al, se concluye que el instrumento es aceptable, es decir si puede ser aplicado nuevamente. En la revisión de los registros de calificaciones de los cursos 2008, 2009 y 2010 comprobamos la secuencia de las falencias verificadas en el pre universitario de la Carrera de Electrónica y Telecomunicaciones. Nos remitimos al año académico 2008, donde encontramos la siguiente novedad, Estudiantes inscritos en el pre -universitario en la carrera de Electrónica y Telecomunicaciones año 2008.

En el periodo académico 2008 - 2009 de un total de 80 estudiantes inscritos en la Carrera de electrónica y Telecomunicaciones, 49 estudiantes aprobaron y 31 estudiantes resultaron reprobados, lo que significó un porcentaje significativo en un proceso donde se requiere calidad. En la siguiente tabla se podrá observar los estudiantes inscritos en el pre -universitario en la carrera de Electrónica y Telecomunicaciones año 2009.

En el periodo académico 2008 - 2009 de un total de 80 estudiantes inscritos en la Carrera de electrónica y Telecomunicaciones, 49 estudiantes aprobaron y 31 estudiantes resultaron reprobados, lo que significó un porcentaje significativo en un proceso donde se requiere calidad. Se podrá observar los estudiantes inscritos en el pre -universitario en la carrera de Electrónica y Telecomunicaciones año 2009. En el periodo académico 2010 - 2011 primer periodo de un total de 178 estudiantes inscritos en la Carrera de electrónica 
y Telecomunicaciones, 52 estudiantes aprobaron, 53 estudiantes reprobaron y se obtuvieron 73 desertores, de lo que se puede deducir que 126 estudiantes reprobaron el pre universitario significando casi las tres cuartas partes de los inscritos, este fue el motivo que originó el análisis de las estrategias didácticas que se aplicaron en los tres periodos académico, lo que infiere un bajo rendimiento de los estudiantes y que se expresa en los registros de calificaciones de mencionados periodos.

La encuesta es una técnica de adquisición de información de interés sociológico, mediante un cuestionario previamente elaborado, a través del cual se puede conocer la opinión o valoración del sujeto seleccionado en una muestra sobre un asunto dado.

\section{CONCLUSIONES}

Por lo tanto ésta técnica fue dirigida a estudiantes del pre universitario, a estudiantes del primero, segundo y tercer nivel de la universidad en la asignatura de matemáticas, así como también a profesores de la carrera. La misma que antes de aplicar las encuestas se realizó una explicación detallada del tema que se investigaba, las estrategias que se iban a seguir y las actividades que planteamos a través de la propuesta, una vez concluida la etapa se procedió a realizar la encuesta aplicando preguntas cerradas y abiertas que nos permitan obtener la mayor información posible.

Se puede determinar que de los estudiantes encuestados en su mayoría coincidieron que los aspectos que no le han satisfecho durante los estudios realizados son: La metodología de enseñanza aplicada no es muy adecuada 
con una frecuencia de 46, la siguiente frecuencia significativa determinó que hay pocas actividades prácticas, y con porcentajes de un dígito se tienen los exámenes finales y la falta de medios de enseñanzas entre otros.

Dentro de los estudiantes encuestados se encontró que en su mayoría los profesores, solo a veces explican con suficiente claridad la asignatura, mientras que una cuarta parte de ellos opinan que si explican, y un porcentaje menor opinaron que no reciben la explicación necesaria en las asignaturas. Por lo que se puede inferir que el profesor no tiene pedagogía precisa para impartir la asignatura de matemáticas lo que incidirá en el bajo rendimiento de los estudiantes del pre- universitario.

El resultado de la encuesta muestra que un tercio de los estudiantes cree que la formación en el colegio de la asignatura de matemáticas es regular, mientras que otro porcentaje similar opina lo que su formación es buena, mientras que una tercera proporción opina que es insuficiente y muy buena.

Cuando se inquirió a los estudiantes; considera que el docente busca terminar el plan académico o que el estudiante aprenda? 43 respondieron que ambas, 31 estudiantes indicaron que se busca terminar el plan académico y un porcentaje menor que el estudiante aprenda. De lo que se infiere que la consigna que tiene cada docente es que "debe terminar el plan académico y que el estudiante aprenda" ellos piensan que si esta consigna se cumpliera en cada periodo de clase no hubieran alumnos reprobados.

En la encuesta realizada a los profesores se obtuvo como resultado que una parte es Ing. en Electrónica y Telecomunicaciones, otro porcentaje en Ing. en Sistemas, y otro porcentaje coinciden en Ing. en Petróleo y especialidad es de Ing. Mecánico. 
La evaluación de desempeño académico es un nuevo instrumento que está establecido en el art.155 de la LOES (Ley Orgánica de Educación Superior) del 12 de octubre del 2010 que dice, Los profesores de las instituciones de educación superior serán evaluados periódicamente en su desempeño académico, por ello hemos considerado la prueba de desempeño pedagógico profesional lo que permitirá inferir si los docentes son evaluados permanentemente a través de una evaluación áulica y por ende establecer su buen desempeño.

\section{REFERENCIAS}

ALBERT, M. (2007). LA investigación educativa. Madrid. Mc Graw Hill

ARANDA, A. (2007). Planificación Estratégica Educativa. 2 $2^{\text {da }}$ Edición. Quito. Ediciones Abya-Yala

Barros-Bastidas, C., \& Turpo, O. (2020). La formación en investigación y su incidencia en la producción científica del profesorado de educa- ción de una universidad pública de Ecuador. Publicaciones, 50(2), 167-185. doi:10.30827/publicaciones.v50i2.13952

BERNAL, C. (2010). Metodología de la Investigación. 3 ${ }^{\text {era }}$ Ed. México. Prentice Hall.

CASTILlO, M. (1996). Estudio sobre el grado de satisfacción de los Estudiantes de la Universidad de los Andes. Bogotá, Colombia, http://www.uniandes.edu.co/boletin/historico/noviembre96

DEL CID et al. (2007) Investigación Fundamentos y Metodología, México, Prentice Hall. 
ECUADOR, (2010), "Ley Orgánica de Educación Superior de Ecuador", Registro Oficial No 298. octubre 12 de 2010. Quito-Ecuador.

HAIR, J. (2010), et al. Investigación de Mercados. $4^{\text {ta }}$ Edición. México. Mc Graw Hill.

HERNANDEZ S. R., FERNANDEZ C. CARLOS, BAPTISTA L. MARÍA : (2010) Metodología de la Investigación, The McGraw-Hill, México.

HERNÁNDEZ SAMPIERI, et al. (2007). Fundamentos de Metodología de la Investigación. México. Prentice Hall.

INEC, (2006) Acceso a la educación en el Ecuador. Análisis de los resultados de la encuesta de condiciones de vida ECV. 2005-2006

INFORME: Educación Superior en Iberoamérica Capítulo Ecuador. Guayaquil, julio de 2006.

JANY, J. (2009). Investigación Integral de Mercados. 4ta Edición. México. Mc Graw Hill.

Lemus, Edel and von Feigenblatt, Otto Federico and Orta, Miguel and Rivero, Orlando, Starbucks Corporation: Leading Innovation in the 21st Century (April 8, 2015). Journal of Alternative Perspectives in the Social Sciences, 7(1), 23-38, 2015, Available at SSRN: https://ssrn.com/abstract=2638468

von Feigenblatt, Otto Federico, The Importance of Historical Heritage and the Fallacy of the Cancel Movement: International Case Studies (May 23, 2020). Journal of Alternative Perspectives in the Social Sciences 
(2020) Volume 10 No 3, 483-492, Available at SSRN: https://ssrn.com/abstract=

von Feigenblatt, Otto Federico, JAPÓN Y EL COVID-19 DESDE UN PUNTO DE VISTA SOCIOCULTURAL (Japan and COVID-19 from a Socio-cultural Perspective) (June 1, 2020). Revista Observatorio Iberoamericano de la Economía y la Sociedad del Japón (febrero 2020)., Available at SSRN: https://ssrn.com/abstract=3663848

von Feigenblatt, Otto Federico, The Significance of the Shang Period for the Contemporary Construction of Chinese Cultural Identity (April 10, 2020). Journal of Business and Entrepreneurial Studies, 4(2), 309318. , Available at SSRN: https://ssrn.com/abstract=

UNESCO. "Evolución Reciente de la Educación en América Latina". Primera parte, página 17. Santiago de Chile. 